Proyecciones

Vol. 21, No 1, pp. 9-19, May 2002.

Universidad Católica del Norte

Antofagasta - Chile

\title{
ASYMPTOTIC EQUILIBRIUM FOR CERTAIN TYPE OF DIFFERENTIAL EQUATIONS WITH MAXIMUM*
}

\author{
PATRICIO GONZÁLEZ \\ Universidad Arturo Prat - Chile \\ and \\ MANUEL PINTO \\ Universidad de Chile - Chile
}

\begin{abstract}
In this work we obtain asymptotic representations for the solutions of certain type of differential equations with maximum. We deduce the asymptotic equilibrium for this class of differential equations.
\end{abstract}

1991 Math. Subject Classification: 34A20

Keywords: Differential equations with maximum, asymptotic representation, asymptotic equilibrium, Banach fixed point theorem.

*This research was supported by FONDECYT Grant \# 8990013 


\section{Introduction}

Differential equations with maximum arise naturally when solving practical problems, in particular, in those which appear in the study of systems with automatic regulation. A classical example is that of an electric generator. In this case, the mechanism becomes actived when the maximum voltage variation that is permited is reached in an interval of time $I_{t}=[t-h, t]$, with $h$ a positive constant.The equation which describes the actioning of this regulator has the form

$$
V^{\prime}(t)=-\delta V(t)+p \max _{s \in I_{t}} V(s)+F(t)
$$

where $\delta$ and $p$ are constants that are determined by the caracteristic of the system, $V(t)$ is the voltage and $F(t)$ is the effect of the perturbation that appears associated to the change of voltage [2].

Much work on these equations has been carried out in the last three decades. We mention the work in [1-2] and $[8,11]$.

We study differential equations with maximum of the form

$$
\left\{\begin{array}{lll}
x^{\prime}(t)=f\left(t, x(t), \max _{u \in I_{t}} x(u)\right), & & \text { with } t \in I \\
x(t)=\varphi(t), & & \text { with } t \in[-h, 0]
\end{array}\right.
$$

where $I=[0, b)$ and the possibility that $b$ be infinity is not excluded. In addition, $f$ is a real - valued continuous function defined on $I \times R \times R$.

We denote by $\|\varphi\|$ the norm

$$
\|\varphi\|=\max \{|\varphi(t)| / t \in[-h, 0]\} \text {. }
$$

Definition 1. A differential equation with maximum (1.1) as above has the property of asymptotic equilibrium if:

1. Every solution $x(t)$ of (1.1) with initial condition $x(t)=$ $\varphi(t)$, for all $t \in[-h, 0]$, is defined for all $t \geq-h$ and there exists $\xi \in R$ which satisfies

$$
\lim _{t \rightarrow \infty} x(t)=\xi
$$


2. For all $\xi \in R$, there exists a solution $x(t)$ of (1.1), which is defined on the interval $[-h, \infty)$ and verifies $(2.1)$.

Our main result asserts, under certain hypotheses on the function $f$, equation (1.1) has asymptotic equilibrium. The techniques used in the proof are based on an inequality of Gronwall - Bellman type and succesive aproximations. The method used is analogous to those of $[3-10]$.

Our principal results are applied to automatic control problems described by nonlinear equations of the type

$$
V^{\prime}(t)=-\delta V(t)+p \max _{s \in I_{t}} V(s)+F(t, V(t)) .
$$

\section{Main Results}

In this paragraph we prove theorems about asymptotic behavior and boundedness of the solutions of equation (1.1). We first recall some basic inequality of Gronwall - Bellman type which we use in the study of the differential equation with maximum (1.1).

Lemma 1. Let $p, x$ be continuous and nonnegative functions on $[0, b)$ and $\varphi_{[-h, 0]}$ a nonnegative continuous function. If the inequality

$$
x(t) \leq x(0)+\int_{0}^{t} p(s) \max _{u \in I_{t}} x(u) d s
$$

holds, for all $t \in[0, b)$, with $x(t)=\varphi(t)$ for all $t \in[-h, 0]$, then

$$
x(t) \leq\|\varphi\| e^{\int_{0}^{t} p(s) d s}
$$

for all $t \in[0, b)$. 
Theorem 1. Suppose that $f$ satisfies the following hypotheses:

$\left[H_{0}\right] \quad f$ is continuous on $I \times R \times R$, where $I=[0, \infty)$.

$\left[H_{1}\right]$ There exist $\lambda$ and $\beta$ integrable functions on $I$ such that, for all $(t, x, y) \in I \times R \times R$, we have

$$
|f(t, x, y)| \leq \lambda(t)(|x|+|y|)+\beta(t)
$$

Then, every solution $x(t)$ with $x(t)=\varphi(t)$, for all $t \in[-h, 0]$, is defined on $[-h, \infty)$ and satisfies (1.2) for some $\xi \in R$. In addition, we have $\mathrm{x}(t)=\xi+O\left(\int_{t}^{\infty}[\lambda(s)+\beta(s)] d s\right)$

Proof. If $x(t)$ is a solution of equation (1.1) such that $x(t)=\varphi(t)$, for all $t$ in $[-h, 0]$, and defined on a subinterval $J=[-h, T)$ of $[-h, \infty)$, then

$$
x(t)=x(0)+\int_{0}^{t} f\left(s, x(s), \max _{u \in I_{s}} x(u)\right) d s,
$$

for all $t$ in $[0, T)$. Therefore, for all $t$ in $[0, T)$, we have

$$
\begin{aligned}
|x(t)| & \leq|x(0)|+\int_{0}^{t}|\lambda(s)|\left(|x(s)|+\left|\max _{u \in I_{s}} x(u)\right|\right) d s+\int_{0}^{t} \beta(s) d s \\
& \leq|x(0)|+\int_{0}^{\infty} \beta(s) d s+\int_{0}^{t} 2 \lambda(s) \max _{u \in I_{s}}|x(u)| d s .
\end{aligned}
$$

By lemma 1,

$$
|x(t)| \leq\left[\|\varphi\|+\int_{0}^{\infty} \beta(s) d s\right] e^{\int_{0}^{t} 2 \lambda(s) d s} \leq\left[\|\varphi\|+\int_{0}^{\infty} \beta(s) d s\right] e^{\int_{0}^{\infty} 2 \lambda(s) d s}
$$

for all $t$ in the interval $J$, which shows that $x(t)$ is bounded in the interval J. Therefore, the left limit $x(T-0)$ exists, when $t$ tends to $T$. Since the initial value problem

$$
y^{\prime}(t)=f\left(t, y(t), \max _{u \in I_{t}} y(u)\right)
$$

with initial condition $y(t)=x(t)$ in $[T-h, T)$ and where $y(T)=$ $x(T-0)$ has a local solution, we conclude that it is possible to extend 
$x$ beyond $T$. This shows that solution $x(t)$ of equation (1.1) is defined on $[-h, \infty)$ and there exists a constant $M$ such that $|x(t)| \leq M$, for all $t \in[-h, \infty)$.

Since $f\left(s, x(s), \max _{u \in I_{s}} x(u)\right)$ is integrable on $I$, from equation (2.2) it follows that

$$
x(t)=x(0)+\int_{0}^{\infty} f\left(s, x(s), \max _{u \in I_{s}} x(u)\right) d s-\int_{t}^{\infty} f\left(s, x(s), \max _{u \in I_{s}} x(u)\right) d s .
$$

Let $\xi$ denote the real number given by

$\mathrm{x}(0)+\int_{0}^{\infty} f\left(s, x(s), \max _{u \in I_{s}} x(u)\right) d s$, then, we have

$$
\begin{aligned}
|x(t)-\xi| & \leq \int_{t}^{\infty} \lambda(s)\left(|x(s)|+\max _{u \in I_{s}}|x(u)|\right) d s+\int_{t}^{\infty} \beta(s) d s \\
& \leq 2 M \int_{t}^{\infty} \lambda(s)+\int_{t}^{\infty} \beta(s) d s
\end{aligned}
$$

thus,

$$
\mathrm{x}(\mathrm{t})=\xi+O\left(\int_{t}^{\infty}[\lambda(s)+\beta(s)] d s\right) .
$$

Theorem 2. If $f$ satisfies conditions

$\left[H_{0}\right] \quad f$ is continuous on $I \times R \times R$ and $f(t, 0,0)$ is integrable on $I$, where $I=[0, \infty)$.

$\left[H_{2}\right]$ There exists a positive and integrable function $\mu$ on $I$ such that, for all $\left(t, x_{1}, y_{1}\right),\left(t, x_{2}, y_{2}\right)$ in $I \times R \times R$, we have

$\left|f\left(t, x_{1}, y_{1}\right)-f\left(t, x_{2}, y_{2}\right)\right| \leq \mu(t)\left(\left|x_{1}-x_{2}\right|+\left|y_{1}-y_{2}\right|\right)$ and there exist a nonnegative real constant $K$ such that, for all $s \in[0, \infty)$

$$
\mu(s) \leq K \mu(s-h)
$$

Then for all $\xi \in R$ there exists a solution $x(t)$ of (1.1) which is defined on $[-h, \infty)$ and verifies (1.2). In addition, we have 


$$
x(t)=\xi+\int_{t}^{\infty} f(s, 0,0) d s+O\left(\int_{t}^{\infty} \mu(s) d s\right) .
$$

Proof. Let $B$ denote the Banach space of the real bounded functions $g$ defined on $[-h, \infty)$ with the norm defined by

$\|g\|=\{\sup |g(t)| / t \in[-h, \infty)\}$.

Consider the operator $T: B \rightarrow B$ defined by

$\mathrm{T}(\mathrm{x})(\mathrm{t})=\xi-\int_{t}^{\infty} f\left(s, x(s), \max _{u \in I_{s}} x(u)\right) d s$ for all $t \geq 0$, and by $\mathrm{T}(\mathrm{x})(\mathrm{t})=\xi$, for all $t \in[-h, 0]$.

In order to prove that $T: B \rightarrow B$ has a fixed point, we must show that there exists an integer $n$ such that $T^{n}$ is a contractive operator. For this, it suffices to show that, for all $x, y$ in $B$ and for all positive integer $n$, we have

$$
\left|\left(T^{n} y\right)(t)-\left(T^{n} x\right)(t)\right| \leq \frac{1}{K n !}\left(\int_{t-h}^{\infty} 2 K \mu(s) d s\right)^{n}\|y-x\| .
$$

We proceed by induction.

First, we verify that the inequality is true for $n=1$.

$$
\begin{aligned}
& |(T y)(t)-(T x)(t)| \\
= & \left|\int_{t}^{\infty}\left(f\left(s, y(s), \max _{u \in I_{s}} y(u)\right)-f\left(s, x(s), \max _{u \in I_{s}} x(u)\right)\right) d s\right| \\
\leq & \int_{t}^{\infty} \mu(s)\left\{|y(s)-x(s)|+\max _{u \in I_{s}}|y(u)-x(u)|\right\} d s \\
\leq & \int_{t}^{\infty} 2 \mu(s) \max _{u \in I_{S}}|y(u)-x(u)| d s \\
\leq & \left(\int_{t}^{\infty} 2 \mu(s) d s\right)\|y-x\| \\
\leq & \frac{1}{K 1 !}\left(\int_{t-h}^{\infty} 2 K \mu(s) d s\right)\|y-x\|
\end{aligned}
$$


Next, if the formula were true for $n-1$, then

$\left|\left(T^{n} y\right)(t)-\left(T^{n} x\right)(t)\right|$

$=\mid \int_{t}^{\infty}\left(f\left(s,\left(T^{n-1} y\right)(s), \max _{u \in I_{s}}\left(T^{n-1} y\right)(u)\right)-\right.$

$\left.f\left(s,\left(T^{n-1} x\right)(s), \max _{u \in I_{s}}\left(T^{n-1} x\right)(u)\right)\right) d s$

$\leq \int_{t}^{\infty} \mu(s)\left\{\left|\left(T^{n-1} y\right)(s)-\left(T^{n-1} x\right)(s)\right|+\right.$

$\left.\max _{u \in I_{s}}\left(T^{n-1} y\right)(u)-\left(T^{n-1} x\right)(u)\right\} d s$

$\leq \int_{t}^{\infty} 2 \mu(s) \max _{u \in I_{s}}\left|\left(T^{n-1} y\right)(u)-\left(T^{n-1} x\right)(u)\right| d s$

$\leq \int_{t}^{\infty} 2 \mu(s) \frac{1}{K(n-1) !}\left(\int_{t}^{\infty} 2 K \mu(\tau) d \tau\right)^{n-1}\|y-x\| d s$

$\leq \int_{t}^{\infty} 2 K \mu(s-h) \frac{1}{K(n-1) !}\left(\int_{t}^{\infty} 2 K \mu(\tau) d \tau\right)^{n-1}\|y-x\| d s$

$\leq \frac{1}{K n !}\left(\int_{t}^{\infty} 2 K \mu(s) d s\right)^{n}\|y-x\|$.

Since $\mu(t)$ is integrable on $I$, we can find a positive integer $n$ such that the operator $T^{n}$ is a contraction. Therefore, there exists a fixed point $x$ of $T$, and then $\mathrm{x}(\mathrm{t})=\xi-\int_{t}^{\infty} f\left(s, x(s), \max _{u \in I_{s}} x(u)\right) d s$ for all $t \geq 0$, and $\mathrm{x}(\mathrm{t})=\xi$, for all $t \in[-h, 0]$, i. e. $x(t)$ is a solution of equation (1.1) such that $x(t)=\varphi(t)$, for all $t$ in $[-h, 0]$.

Since $\left|x(t)-\xi-\int_{t}^{\infty} f(s, 0,0) d s\right| \leq K \int_{t}^{\infty} \mu(s) d s$ we can see that (2.3) is verified.

Example 1 : Consider the differential equation with maximum

$$
\left\{\begin{array}{c}
x^{\prime}(t)=\frac{2}{(1+t)^{2}} \max x(u) \\
x(t)=e^{-2}, \text { for } t \in[-h, 0]
\end{array}\right.
$$

We have that $f(t, x, y)=\frac{2}{(1+t)^{2}} y$ is a real-valued, continuous, integrable function on $I \times R \times R$ and

$$
|f(t, x, y)|=\left|\frac{2}{(1+t)^{2}} \cdot y\right| \leq \frac{2}{(1+t)^{2}}(|x|+\mid y) \mid \text {. }
$$


The function $\lambda(t)=\frac{2}{(1+t)^{2}}$ is continuous and integrable on I . Thus $f$ satisfies conditions $H_{0}$ and $H_{1}$ and by Theorems 1 and 2, every solution $x(t)$ is defined on $[-h, \infty)$ and verifies

$$
x(t)=\xi+O\left(\int_{t}^{\infty} \lambda(s) d s\right)=\xi+O\left(\frac{2}{1+t}\right)
$$

and reciprocally for any $\xi \in R$ there exists a solution $x$ defined on $[-h, \infty]$ and satisfying asymptotic formula $(2.5)$.

In this example, the function $\mathrm{x}(\mathrm{t})=\left\{\begin{array}{c}\exp \left(-\frac{2}{1+t}\right), \quad \text { for } t \in I \\ \exp (-2), \quad \text { for } t \in[-h, 0]\end{array}\right.$ is an explicit solution of the differential equation which in effect satisfies formula (2.5).

Example 2. In the differential equation with maximum

$$
\left\{\begin{array}{c}
x^{\prime}(t)=\frac{2+\sin t}{1+t^{2}} x(t)+\frac{2}{(1+t)^{2}} \max _{u \in I_{t}} x(u) \\
x(t)=\sigma
\end{array}\right.
$$

the function $f(t, x, y)=\frac{2+\sin t}{1+t^{2}} x+\frac{2}{(1+t)^{2}} y$ is continuous for $t>-1, x \in R, y \in R$.

Moreover,

$$
\begin{aligned}
&\left|f\left(t, x_{1}, y_{1}\right)-f\left(t, x_{2}, y_{2}\right)\right|=\mu_{1}(t)\left|x_{1}-x_{2}\right|+\mu_{2}(t)\left|y_{1}-y_{2}\right| \\
& \leq \mu(t)\left(\left|x_{1}-x_{2}\right|+\left|y_{1}-y_{2}\right|\right)
\end{aligned}
$$

with $\mu(t)=\max \left\{\mu_{1}(t), \mu_{2}(t)\right\}$, where $\mu_{1}(t)=\frac{|2+\sin t|}{1+t^{2}}$ and $\mu_{2}(t)=\frac{2}{(1+t)^{2}}$.

Since $f$ satisfies conditions $H_{0}, H_{1}$ and $H_{2}$ we have that for every solution $x(t)$ of the given equation, there exists $\xi \in R$ such that $\mathrm{x}(\mathrm{t})=\xi+O\left(\frac{\pi}{2}-\arctan t\right)$.

Conversely, for each $\xi \in R$, there exist a solution $x(t)$ defined on 
the interval $[-h, \infty)$ such that

$$
x(t)=\xi+O\left(\int_{t}^{\infty} \lambda(s) d s\right)=\xi+O\left(\frac{\pi}{2}-\arctan t\right) .
$$

Example 3 : (Application to semilinear electrogenerators) : Consider the differential equation with maximum

$$
\left\{\begin{array}{c}
x^{\prime}(t)=a(t) x(t)+g\left(t, x(t), \max _{s \in I_{t}} x(s)\right), \text { for } t \in[0, \infty) \\
x(t)=e^{\int_{0}^{t} a(s) d s} \xi, \quad \text { for } \quad t \in[-h, 0]
\end{array}\right.
$$

where $a$ is a continuous function on $I$ and $g$ is a continuous function on $I \times R \times R$, such that $\mathrm{f}(\mathrm{t}, \mathrm{x}, \mathrm{y})=\mathrm{e}^{-\int_{0}^{t} a(s) d s} g\left(t, e^{\int_{0}^{t} a(s) d s} x, e^{\int_{0}^{t} a(s) d s} y\right)$ verifies conditions $H_{0}$ and $H_{2}$.

Substitution $x(t)=e^{\int_{0}^{t} a(s) d s} v(t)$ in this equation gives $\mathrm{v}^{\prime}(t)=e^{-\int_{0}^{t} a(s) d s} g\left(t, e^{\int_{0}^{t} a(s) d s} v(t), \max _{s \in I_{t}} e^{\int_{0}^{s} a(\tau) d \tau} v(s)\right)$

By Theorem 2 , there exists a solution $v$ of the differential equation

$$
\begin{aligned}
& \left\{\begin{array}{r}
v^{\prime}(t)=f\left(t, v(t), \max _{s \in I_{t}} v(s)\right) \\
v(t)=\xi, t \in[-h, 0]
\end{array}\right. \\
& \mathrm{v}(\mathrm{t})=\xi+\int_{t}^{\infty} f(s, 0,0) d s+O\left(\int_{t}^{\infty} \mu(s) d s\right) \text { then } \\
& \mathrm{x}(\mathrm{t})=\mathrm{e}_{0}^{\int_{0}^{t} a(s) d s}\left\{\xi+\int_{t}^{\infty} e^{-\int_{0}^{s} a(\tau) d \tau} g(s, 0,0) d s+O\left(\int_{t}^{\infty} \mu(s) d s\right)\right\}
\end{aligned}
$$

If function $a$ is integrable in the interval $I$ then the solutions of the equation are stables. If $\int_{0}^{t} a(s) d s$ tends to $-\infty$ as $t$ approaches infinity then, the solutions of the system are asymptotically stables.

We can apply this result to an automatic control problem described by a nonlinear equation of the type

$$
\left\{\begin{array}{c}
V^{\prime}(t)=-\delta V(t)+p(t) \max _{s \in I_{t}} V(s)+F(t), \text { if } t \in I \\
V(t)=e^{-\delta t} \xi, \text { if } t \in[-h, 0]
\end{array}\right.
$$

where $\delta$ is a positive constant, $p, F$ are continuous and integrable functions on $I$ and there exists a nonnegative real constant $K$, such that, for all $s \in[0, \infty), p(s) \leq K p(s-h)$. We can see there exists a solution 
$V(t)$ of this equation such that the following asymptotic representation is verified $\mathrm{V}(\mathrm{t})=\mathrm{e}^{-\delta t}\left\{\xi+\int_{t}^{\infty} e^{-\delta s} F(s) d s+O\left(\int_{t}^{\infty} p(s) d s\right)\right\}$

\section{References}

[1] N. R. Bantsur and E. P Trofimchuk, Existence and stability of the periodic and almost periodic solutions of quasilinear systems with maxima. Ukrain. Math. J. 6, pp. 747 - 754, (1998).

[2] D. D. Bainov and N. G. Kazakova, A finite difference method for solving the periodic problem for autonomous differential equations with maxima, Math. J. Toyama Univ., 15, pp. 1-13, (1992).

[3] V. H. Cortés and P. González, Levinson's theorem for impulsive differential equations, Analysis 14, pp. 113-125, (1994).

[4] P. González and M. Pinto, Stability Properties of the Solutions of the Nonlinear Functional Differential Systems. J. Math. Anal. Appl. Vol. 181, 2, pp. 562-573, (1994).

[5] P. González and M. Pinto, Asymptotic behavior of impulsive differential equations, Rocky Mountain Journal of Mathematics, 26, pp. 165 - 173, (1996).

[6] P. González and M. Pinto, Asymptotic behavior of the solutions of certain complex differential equations, Differential Equations and Dinamical Systems, 5, pp. 13 - 23, (1997).

[7] J. Guzman and M. Pinto, Global existence and asymptotic behavior of solutions of nonlinear differential equations, J, Math. Anal. Appl. 186, pp. 596 - 604, (1994).

[8] A. D. Myshkis, On some problems of the theory of differential equations with deviating argument, Russ. Math. Surv. 32 (2), pp. 181 - 210, (1977). 
[9] M. Pinto, Asymptotic Integration of System Resulting from a Perturbation of an h-system, J. Math. Anal. Appl. 131, pp. 144-216, (1988).

[10] M. Pinto, Impulsive Inequalities of Bihari Type. Libertas Math. 12, pp. 57-70, (1993).

[11] A. M. Samoilenko, E. P. Trofimchuk and N. R. Bantsur, Periodic and almost periodic solutions of the systems of differential equations with maxima. Proc. NAS Ukraine, pp. 53 - 57 (in Ukrainian), (1998).

Received : December 2001.

\section{Patricio González}

Departamento de Ciencias Físicas y Matemáticas

Universidad Arturo Prat

Avenida Arturo Prat 2120

Casilla 121

Iquique

Chile

email: pgonz@cavancha.cec.unap.cl

and

\section{Manuel Pinto}

Departamento de Matemáticas

Facultad de Ciencias

Universidad de Chile

Casilla 653

Santiago

Chile

email : pintoj@uchile.cl 